\title{
O itinerário terapêutico de famílias de crianças com câncer: dificuldades encontradas neste percurso
}

\section{Como citar este artigo:}

Lima BC, Silva LF, Góes FGB, Ribeiro MTS, Alves LL. 0 itinerário terapêutico de familias de crianças com câncer: dificuldades encontradas neste percurso. Rev Gaúcha Enferm. 2018:39:220180004. doi: https://doi.org/10.1590/19831447.2018.20180004.

\footnotetext{
Universidade Federal Fluminense (UFF). Niterói, Rio de Janeiro, Brasil.

b Universidade Federal Fluminense (UFF). Escola de Enfermagem. Departamento de Enfermagem Materno-infantil e Psiquiátrica. Niterói, Rio de Janeiro, Brasil.

'Universidade Federal Fluminense (UFF). Escola de Enfermagem. Departamento de Enfermagem de Rio das Ostras. Rio das Ostras, Rio de Janeiro, Brasil.
}

\author{
Bruna da Conceição de Lima ${ }^{a}$ \\ Liliane Faria da Silva ${ }^{b}$ \\ Fernanda Garcia Bezerra Góes ${ }^{c}$ \\ Martha Tudrej Sattler Ribeiro ${ }^{\mathrm{a}}$ \\ Luciana Lima Alves ${ }^{\mathrm{a}}$
}

\section{RESUMO}

Objetivo: Conhecer as dificuldades encontradas pelas famílias no itinerário terapêutico de crianças com câncer. Método: Pesquisa descritiva e exploratória, de abordagem qualitativa, realizada de março a novembro de 2016, através de entrevistas semiestruturadas, com 21 familiares de crianças em tratamento oncológico em um hospital universitário federal no Rio de Janeiro, cujos dados foram submetido à análise temática.

Resultados: As dificuldades das famílias perpassam a identificação e a investigação pelos profissionais de saúde dos sinais e sintomas da criança e a passagem dos familiares por diversos serviços de saúde até a confirmação diagnóstica.

Considerações finais: 0 diagnóstico precoce do câncer infantil depende de ações de instituições de saúde e de ensino para a apropriada investigação da doença pelos profissionais, entre eles 0 enfermeiro que atua nas classificações de risco das emergências e na atenção básica, além do adequado funcionamento do sistema de referência e contrarreferência do sistema de saúde.

Palavras-chave: Neoplasias. Criança. Diagnóstico precoce. Diagnóstico tardio. Atenção primária à saúde. Enfermagem pediátrica.

\section{ABSTRACT}

Objective: To know the difficulties faced by families in the therapeutic pathway of children with cancer.

Method: Qualitative, descriptive and exploratory research, with a quantitative approach, performed from March to November 2016, through face-to-face semi-structured interviews, with 21 relatives of children undergoing oncological treatment at a federal university hospital in Rio de Janeiro, whose data were submitted to content analysis.

Results: The difficulties of these families traverse the identification and investigation by health professionals regarding the signs and symptoms of children as well as the passage of relatives through various health services until diagnostic confirmation.

Final considerations: The early diagnosis of childhood cancer depends on actions from health and teaching institutions for the appropriate investigation of the disease by professionals, including the nurse who works with risk classification in emergency departments and in primary care, besides the appropriate operation of the reference and counter-reference system of the health system. Keywords: Neoplasms. Child. Early diagnosis. Delayed diagnosis. Primary health care. Pediatric nursing.

\section{RESUMEN}

Objetivo: conocer las dificultades encontradas por las familias en el trayecto terapéutico de niños con cáncer.

Método: investigación cualitativa, descriptiva y exploratoria, con planteamiento cualitativo, efectuada entre marzo y noviembre de 2016, mediante entrevistas semiestructuradas presenciales, con 21 parientes de niños bajo tratamiento oncológico en un hospital universitario federal en Río de Janeiro, cuyos datos se sometieron al análisis de contenido.

Resultados: las dificultades de las familias atraviesan la identificación e investigación por los profesionales de salud de los signos y síntomas del niño y el pasaje de los parientes por diversos servicios sanitarios hasta la confirmación diagnóstica.

Consideraciones finales: el diagnóstico precoz del cáncer infantil depende de acciones de instituciones de salud y de enseñanza para la apropiada investigación de la enfermedad por los profesionales, incluyendo el enfermero que actúa en las clasificaciones de riesgo de las emergencias y en la atención primaria, además del adecuado funcionamiento del sistema de referencia y contrarreferencia del sistema de salud.

Palabras clave: Neoplasias. Niño. Diagnóstico precoz. Diagnóstico tardío. Atención primaria de salud. Enfermería pediátrica. 


\section{—INTRODUÇÃO}

O câncer no Brasil foi responsável, em 2013, por cerca de 2.800 casos de mortes entre crianças e adolescentes de 0 a 19 anos, ocupando a segunda posição entre os óbitos ocorridos nesta faixa etária, ficando abaixo somente das mortes por causas externas. Para o biênio 2018-2019, no cenário brasileiro, estima-se 12.500 casos novos dessa doença para esse grupo populacional(1)

Quando na infância, o câncer configura-se como uma doença de alta letalidade, possuindo diversas apresentações clínicas devido às diferenças quanto à origem, fatores de risco, tipos histológicos, sítios anatômicos e respostas ao tratamento. Os tumores pediátricos, em geral, são de origem embrionária e apresentam curto período de latência, logo, os fatores ambientais exercem pouca ou nenhuma influência para sua ocorrência, além disso, possuem sintomatologia inespecífica e tendem a ser mais agressivos e de evolução mais rápida. Portanto, a associação dessas características dificultam o diagnóstico e o tratamento oportuno do câncer infantil, fazendo com que a detecção precoce seja imperativa para um bom prognóstico, aumentando, assim, a chance de melhores resultados com menos sequelas ${ }^{(2)}$.

Estudo realizado no Reino Unido destacou que entre os principais sinais e sintomas de alerta identificados nas crianças em consulta na atenção primária, três meses antes do diagnóstico de câncer infantil, destacam-se a cefaleia, linfadenopatias, hepatoesplenomegalia, petéquias e fadi$\mathrm{ga}^{(3)}$. Assim, a partir do surgimento dos primeiros sinais e sintomas de adoecimento até a confirmação diagnóstica precoce ou tardia da doença, existe uma trajetória a ser percorrida pela criança e sua família. Esse caminho, associado às práticas individuais e socioculturais, na tentativa de solucionar os problemas de saúde da criança, é denominado itinerário terapêutico(4).

Estudos sobre itinerários terapêuticos de pacientes em busca de atenção à saúde auxiliam a compreensão do comportamento frente ao cuidado, assim como a forma de funcionamento e utilização dos serviços de saúde. Porém, os caminhos percorridos em busca de cuidados, por vezes, são distintos e não coincidem necessariamente com esquemas ou fluxos pré-determinados pelo sistema de saúde. Deste modo, o trajeto realizado de maneira inadequada pode projetar um diagnóstico tardio, diminuindo as chances de cura e aumentando os riscos de sequelas e óbito(4).

É importante salientar que o itinerário terapêutico tem sido alvo de pesquisas com familiares de crianças e adolescentes em diferentes situações de adoecimento, tais como doença respiratória, diabetes, câncer, entre outras, permitindo a visualização da trajetória da criança e família pelo sistema de saúde e os aspectos sociais e culturais envolvidos no processo de adoecimento ${ }^{(5-7)}$. Contudo, para melhor compreensão desses itinerários, faz-se importante aproximação com os Modelos Explicativos e os subsistemas que compõem o Sistema de Cuidado à Saúde.

Os Modelos Explicativos são as concepções sobre a enfermidade e as formas de tratamento empregadas por todos aqueles engajados no processo clínico e que vão definir qual setor do Sistema de Atenção à Saúde será acionado no processo de cuidado. Sistemas de Atenção à Saúde são constituídos por três setores (subsistemas) que caracterizam práticas de cuidado de naturezas diversas: o profissional, o popular e o familiar ${ }^{(4)}$. Neste estudo, a ênfase foi dada ao subsistema profissional que inclui os profissionais da área da saúde e a rede oficial de assistência à saúde.

Nesse contexto, os enfermeiros devem conhecer o percurso das crianças com câncer e usá-lo como instrumento para a assistência de enfermagem, promoção da saúde, prevenção de agravos e detecção precoce do câncer infantil ${ }^{(5)}$. Contudo, ainda urge a necessidade de uma melhor compreensão dos fatores que podem atrasar o diagnóstico do câncer infantil, no intuito de auxiliar a adoção de estratégias governamentais, institucionais e profissionais que preencham as lacunas deste percurso, de modo que a universalidade, a equidade e a integralidade sejam garantidas.

Diante do exposto, a questão norteadora do estudo foi: Quais as dificuldades encontradas pelas famílias no itinerário terapêutico de crianças com câncer? Para responder esse questionamento, o objetivo do estudo foi conhecer as dificuldades encontradas pelas famílias no itinerário terapêutico de crianças com câncer.

\section{口 MÉTODO}

O presente estudo é resultado de um trabalho de conclusão de curso de graduação(8) em enfermagem, configurando-se como uma pesquisa descritiva e exploratória, de abordagem qualitativa, realizada no ambulatório de quimioterapia de um hospital universitário federal pediátrico localizado no município do Rio de Janeiro.

O cenário da pesquisa é um dos hospitais certificados pelo Ministério da Saúde e Ministério da Educação, pois além das tradicionais atividades de atenção à saúde, promove formação de recursos humanos e pesquisa, o desenvolvimento tecnológico para o sistema de saúde, além de contribuir na formulação de políticas públicas. Além disso, possui a habilitação Unacon, Unidades de Assistência de Alta Complexidade, do Ministério da Saúde, pois reúne condições técnicas, instalações físicas, equipamentos e recur- 
sos humanos adequados à prestação de assistência especializada de alta complexidade para o diagnóstico definitivo e tratamento do câncer, no caso específico, de crianças.

E sendo uma instituição voltada ao atendimento especializado dos problemas de saúde na infância, tem-se constituído, ao longo do tempo, em hospital de referência para atendimento de condições clínicas complexas e raras. Ademais recebe crianças de diferentes regiões do estado do Rio de Janeiro e de outros estados brasileiros, inclusive para o tratamento do câncer infantil, especialmente os casos de leucemia e linfoma.

Os participantes do estudo foram 21 familiares de crianças em tratamento oncológico neste cenário. Os critérios de inclusão dos participantes foram: idade maior ou igual a dezoito anos; e, familiares de crianças em tratamento oncológico que eram responsáveis pelo cuidado direto desde o início dos sintomas. Foram excluídos: familiares de crianças que necessitavam de acompanhantes em tempo integral, impossibilitando o afastamento temporário para a entrevista; familiares de crianças em cuidados paliativos, pois ao falarem sobre o caminho percorrido poderia, de algum modo, ocorrer associação da evolução da doença com as decisões tomadas em seus percursos, neste caso, a participação poderia expor os familiares a sentimentos de culpa ou tristeza.

A coleta de dados se deu por meio de entrevistas semiestruturadas, nos meses de março a novembro de 2016, conduzidas pela primeira autora após treinamento e orientação da professora doutora, segunda autora, experiente em pesquisas qualitativas. Para tal, foi utilizado um roteiro com as seguintes perguntas: $O$ que percebeu de diferente que chamou sua atenção para a saúde da criança? Fale sobre o caminho que você percorreu para buscar ajuda quando percebeu que havia algo diferente com a criança. Quais foram dificuldades que encontraram nesse caminho que percorreu até começar o tratamento?

Os familiares, que atendiam aos critérios de inclusão, foram convidados pessoalmente e esclarecidos quanto à pesquisa e seus objetivos. Após o aceite, as entrevistas foram realizadas pela pesquisadora, em local privado do cenário da pesquisa, sem a presença de outras pessoas. Para registrar precisamente as falas dos participantes, as entrevistas foram gravadas com auxílio de uma mídia digital e posteriormente transcritas na íntegra.

Foi garantido o anonimato dos participantes através da utilização de um código, a saber, a palavra "Entrevista" seguida do número arábico respectivo à sequência de realização (Entrevista 1, 2, 3 e assim por diante). Cumpre destacar que não houve recusas e tampouco desistências quanto à participação o estudo.
A pesquisa seguiu as determinações da Resolução 466/12 do Conselho Nacional de Saúde e foi submetida ao Comitê de Ética em Pesquisa (CEP) do cenário da pesquisa, sendo aprovada com o número do parecer 1.392 .303 e CAAE no 51022115.8.0000.5264. Todos os participantes assinaram um Termo de Consentimento Livre e Esclarecido.

As entrevistas foram analisadas seguindo-se as três fases da análise temática ${ }^{(9)}$ : pré-análise, com leitura flutuante para conhecer o conteúdo do material empírico gerado pelas entrevistas; fase de exploração do material, quando os dados brutos foram transformados em unidades que representavam significados e depois agregados em categorias; fase de tratamento e a interpretação dos resultados, quando foi possível fazer inferências à luz da literatura científica.

Assim, emergiram duas categorias temáticas que versaram sobre o subsistema profissional, abrangendo os profissionais de saúde e a rede de atendimento: o encontro da família com o (des)preparo dos profissionais para o diagnóstico de câncer infantil e a peregrinação da família pelos diversos níveis de atenção à saúde.

\section{口RESULTADOS E DISCUSSÃO}

Quanto aos familiares das crianças, dos 21 participantes, 17 eram mães; 03 eram pais; e 01 era avó; 05 tinham entre 20-30 anos; 14 tinham entre 30-40 anos; e 02 tinham mais de 40 anos. Em relação às 21 crianças, a idade era de 2 a 12 anos de idade; 20 tinham diagnóstico de Leucemia Linfoblástica Aguda (LLA) e 01 de Leucemia Mielóide Aguda (LMA). No que diz respeito ao tempo entre o início dos sinais e sintomas à definição do diagnóstico 02 levaram 02 dias; 01 levou 05 dias; 02 levaram 10 dias; 01 levou 13 dias; 01 levou 15 dias; 03 levaram 01 mês; 03 levaram 02 meses; 04 levaram 03 meses; 02 levaram 04 meses e 02 levaram 06 meses.

\section{O encontro da família com o (des)preparo dos profissionais para o diagnóstico do câncer infantil}

Sobre o caminho percorrido pela família na busca de atendimento, mediante a percepção de algo de diferente com a criança, emergiram depoimentos relativos ao despreparo de profissionais de saúde na suspeição e no diagnóstico do câncer e na realização do encaminhamento à unidade especializada. Por outro lado, alguns familiares relataram o contato com profissionais que realizaram oportunamente a confirmação diagnóstica e a adequada referência à unidade de tratamento do câncer. 
Na procura por assistência, a partir da ocorrência dos primeiros sinais e sintomas do câncer infantil, como hematomas, dificuldade para andar, febre, dor, dentre outros, os familiares declararam que as crianças receberam diagnósticos e tratamentos errôneos, pela não associação do quadro apresentado pela criança ao câncer, além de serem submetidas a diferentes procedimentos, bem como vivenciarem inúmeras idas e vindas a diferentes serviços de saúde; o que prolongou o tempo para a confirmação definitiva da doença.

Eu levava ela para emergência, mas não adiantou de nada, porque eles falavam que era virose. [...] Ficava levando ela em hospital e nada. Só depois que descobriu, mas mesmo assim só porque ela parou de andar e antes falaram que era leptospirose. (Entrevista 12)

Antes de eu chegar na ortopedista, que fez exame que deu reumatismo, ele passou muito por emergências e eles sempre diziam que era uma sinovite transitória no quadril e que passaria. Diziam que ele poderia ter caído de mau jeito ou alguma coisa. (Entrevista 14)

Ele teve dor no ombro e levamos a uma consulta normal [de rotina]. Falaram que era luxação. Levei no ortopedista que fez raio x e também não deu nada e aí começou a febre e os médicos acharam que era uma virose, alguma coisa assim, tipo dengue. [...]. Na terceira vez que levei ele na emergência, a médica resolveu internar porque viu que o estado dele estava piorando e internou como pneumonia. (Entrevista 6)

A pediatra disse que ele tinha hematomas porque estava se batendo muito, estava se machucando. [...] Um mês depois fez febre, febre baixinha, levei na pediatra de novo, aí ela disse que era crise alérgica. Depois, além da febre ele deu umas bolas roxas dentro da boca, vários hematomas. E eu levei e ela disse que ele podia estar fazendo alergia ao spray da bronquite porque ele tem asma. (Entrevista 17)

O fato de o câncer nas crianças apresentar sintomas vagos e inespecíficos, por vezes mal interpretados, por simularem condições comuns e inofensivas na infância, pode aumentar o intervalo entre o início da sintomatologia e o diagnóstico ${ }^{(10)}$, como evidenciado no estudo. Dessa forma, enquanto a criança não apresenta um quadro mais grave da doença, a obtenção do diagnóstico torna-se mais difícil.

Logo, os achados evidenciam que o atendimento que algumas crianças receberam, com a falta de reconhecimento da sintomatologia sugestiva do câncer, está confli- tante com o que a literatura recomenda. Há que se considerar que a ênfase atual para o câncer infantil requer que o processo de suspeita e investigação seja precoce, a fim de que esse paciente se beneficie de um tratamento com todo potencial e eficácia necessários, objetivando, assim, bons índices de cura e redução das sequelas da doença(2). Portanto, o encaminhamento adequado, oportuno e informativo da atenção primária para a secundária é uma condição nodal(10), o que não foi prevalente nas falas dos familiares do estudo.

Estudo realizado no Reino Unido mostrou que crianças com câncer foram atendidas na atenção primária, antes do diagnóstico, com maior frequencia que as crianças em geral $^{(3)}$. Portanto, os profissionais de saúde, entre eles o enfermeiro, precisam estar atentos à presença de sinais e sintomas de alerta e a busca frequente de crianças pelos serviços de saúde, pois esses são fatores associados a uma maior probabilidade do câncer infantil.

Uma das crianças apresentou recidiva da doença, e ainda que submetida ao tratamento oncológico anteriormente, também houve demora quanto ao diagnóstico de sua recaída.

Quando ele teve a segunda vez [recaída da leucemia], foi no testículo e eu fiquei um mês e pouco cuidando como se fosse uma caxumba. Fomos na UPA de lá [Macaé] e em um urologista. Ele fez exames, ultrassom e ele disse que era da caxumba. Daí mostrei os exames a um médico daqui do Rio [...]. Na hora que olhou falou: Não, não é caxumba, isso é a leucemia dele que está aí! (Entrevista 20)

Desde que foi possível alcançar o sucesso no tratamento terapêutico do câncer infantil, é sabido que o diagnóstico precoce e o tratamento em unidades de referência são fundamentais para a obtenção da cura e da qualidade de vida. No entanto, no Brasil, casos de câncer com potencial de cura ainda são identificados em estágios avançados, o que impacta negativamente na mortalidade ${ }^{(2)}$.

Dessa forma, é imperativa a necessidade de registros detalhados, mediante uma boa coleta de dados e exame físico minucioso, particularmente em casos que envolvem crianças com sintomas vagos e persistentes, o que de fato pode ajudar na garantia do diagnóstico em tempo adequado ${ }^{(10-11)}$

Portanto, nesse contexto, o enfermeiro possui a capacidade de prestar uma assistência eficaz, por meio do processo de enfermagem, identificando problemas de saúde na criança, favorecendo, inclusive, o diagnóstico precoce do câncer infantil. Sabe-se que o itinerário terapêutico é envolvido pelo planejamento e estratégias que 
buscam sanar os problemas envolvendo a saúde dos pacientes, sendo imprescindível a atuação da enfermagem neste processo ${ }^{(12)}$.

A persistência dos sinais e sintomas e a não resolução do problema fez com que as famílias fossem persistentes, levando seus filhos a diferentes serviços de saúde para que a adequada investigação diagnóstica fosse realizada. Ademais, houve relatos de que consultas foram finalizadas sem a solicitação de exames complementares.

Comecei a levar ele na emergência, os médicos pediam para baixar a calça dele e falavam que era uma batida e passavam remédio. Nunca encostaram no meu filho para examinar, nunca pediram exame de sangue [...]. Se eles tivessem examinado, dava para ver. (Entrevista 2)

A gente levou ele na emergência. O pediatra atendeu, mas não fez nenhum exame na primeira vez não. Falou que ele estava com a saúde muito boa e que ele não tinha nada. (Entrevista 8)

Vale enfatizar que o histórico detalhado proporciona diagnósticos adequados e terapias assertivas, além de possibilitar uma relação humanizada entre o profissional e o paciente. Quando a anamnese não é realizada, ocorre muito rapidamente ou as queixas do paciente não são escutadas atentivamente, pode haver comprometimento na qualidade do tratamento, devido, inclusive, o atraso na confirmação diagnóstica ${ }^{(11)}$.

Um familiar relatou ainda abordagem inadequada durante o atendimento em um serviço de saúde.

Uma médica que atendeu na emergência pediu para ver e começou a brigar comigo, dizendo que não era caso de emergência e que eu estava atrapalhando o serviço dela. (Entrevista 2)

Com base nesse relato, é possível observar que uma comunicação ineficiente ou uma abordagem inadequada podem acarretar uma série de conflitos, inseguranças e até interferir no diagnóstico precoce do câncer infantil. Sendo assim, a comunicação, mediada por uma escuta atentiva, é uma importante prática do cuidado humanizado no que se refere ao contato estabelecido junto aos familiares das crianças.

$\mathrm{Na}$ tentativa de solucionar efetivamente o problema de saúde da criança, os familiares se tornaram protagonistas dentro do caminho, buscando outros serviços de saúde ou ameaçando entrar na justiça, uma vez que acreditam que o tratamento da criança não estava adequado.
Minha neta estava internada e saiu de lá sem diagnóstico. Ameacei ir ao fórum, abrir uma liminar para eles fazerem alguma coisa. [...] (Entrevista 1)

Ele parou de andar, não ficava nem mais em pé. Aí eu fiquei doida, corri para um hospital grande para salvar meu filho. Fiquei internada com ele lá um mês e também não descobriram nada. Ai foram fazer um exame na barriga dele e viram que o baço dele estava alterado. (Entrevista 21)

Dados de outra pesquisa mostraram que algumas vezes diversos serviços de saúde têm organizado seus processos de trabalho com foco no procedimento e não no paciente, deixando-se de lado a escuta qualificada e priorizando a realização de exames. $O$ atendimento das necessidades de saúde quando não é efetivo reflete em novas ações, visto que as famílias e o próprio paciente são levados a buscar outros caminhos e serviços que poderão thes oferecer acesso ao atendimento de sua demanda ${ }^{(12)}$.

Em contrapartida, houve casos em que as famílias se depararam com profissionais mais preparados, pois durante o exame físico ou avaliação de exames suspeitaram da possibilidade de um câncer.

Uma pediatra [primeiro atendimento em hospital privado] atendeu e não foi detectado logo de imediato, o exame deu tudo bem. O que chamou atenção da médica foi que o fígado estava aumentado, ele internou e 4 dias depois já tinha diagnóstico de leucemia. (Entrevista 3)

A médica [primeiro atendimento na emergência] examinou ele e viu que o baço dele estava grande e o exame de sangue dele estava com tudo alterado. Daí eles falaram comigo e conseguiram uma vaga aqui [centro de tratamento]. Aqui fizeram a lâmina, o exame da medula e diagnosticou que ele tinha leucemia. (Entrevista 9)

Fizeram exame de sangue [na UPA] e transferiram para outro hospital. Lá eles me disseram que estava com leptospirose, mas desconfiaram que podia ser leucemia também [...]. Me transferiram para cá onde teve o diagnóstico. (Entrevista 18)

Os dados apontam que para o diagnóstico precoce do câncer infantil e um bom prognóstico da doença é essencial que os profissionais de saúde, incluindo médicos e enfermeiros, estejam preparados para o primeiro atendimento de uma possível criança nessa situação, já que na maioria das vezes o primeiro atendimento não é realizado por um especialista. Portanto, é fundamental que 
as instituições de saúde e de ensino capacitem o pessoal da saúde tendo por base as necessidades de saúde dos indivíduos e das populações nos variados níveis de atenção à saúde, vislumbrando a construção da integralidade do cuidado ${ }^{(13)}$.

No que tange à enfermagem, as Diretrizes Curriculares Nacionais (DCN) preconizam a formação de um enfermeiro generalista e humanista que atue sobre a realidade social e atenda às necessidades da população com compromisso à saúde integral do ser humano e responsabilidade. Assim, o perfil esperado apresenta a capacidade de reconhecer e intervir sobre os variados problemas e situações do processo saúde-doença ${ }^{(14)}$.

Dessa forma, o enfermeiro, nos diferentes níveis de atenção à saúde, da atenção primária à terciária, incluindo o acolhimento com classificação de risco nos serviços de emergência, deve estar capacitado e apto para atender as crianças e suas famílias e promover um cuidado integral. Sua atuação eficiente deve incluir a capacidade de reconhecer os sinais e sintomas sugestivos do câncer infantil, contribuindo, assim, para a suspeição precoce, com o devido encaminhamento da criança ao médico especialista que realizará a confirmação diagnóstica.

\section{A peregrinação da família pelos diversos níveis de atenção à saúde}

Quando os primeiros sinais e sintomas do câncer na criança são observados pela família, inicia-se uma caminhada, muitas vezes árdua, por diversos serviços de saúde, o que representa uma peregrinação na busca pelo diagnóstico e tratamento.

Uma família iniciou sua trajetória em Unidades Básicas de Saúde (UBS), como preconiza o Sistema Único de Saúde (SUS), entretanto, por não obter a resolução do problema, a família buscou outros níveis de atenção.

Levamos ele em alguns postos de saúde em Caxias, uns diziam que era apenas uma anemia forte, outros diziam que não era nada. Aí levamos duas vezes, no mesmo dia, na emergência e na segunda vez fez exames e a médica encaminhou a gente pra cá [centro de tratamento], pra ter um diagnóstico completo (Entrevista 8).

O relato do familiar 8 aponta que mesmo procurando a atenção primária, como primeiro atendimento, a criança não recebeu o diagnóstico nem foi encaminhada para uma unidade de saúde especializada. Com a manutenção dos sintomas, a criança foi levada para o segundo nível de atenção, que compreende as emergências, para então ser referenciada ao terceiro nível para receber o diagnóstico e tratamento devidos.

Sabe-se que a UBS é a principal porta de entrada e centro de comunicação com toda a rede de atenção à saúde. Nesse sentido, quando a família percebe algo de errado com a criança, muitas vezes é neste tipo de unidade que se inicia a investigação do quadro clínico. Entretanto, essas famílias podem percorrer um longo caminho até alcançar uma unidade especializada de tratamento(15). Pesquisa que estudou o itinerário terapêutico de famílias de crianças com doenças respiratórias também identificou insuficiências na UBS que implicaram em internações por condições que poderiam ter sido evitadas $s^{(6,15)}$.

Nessa perspectiva, o enfermeiro que atua na atenção primária precisa estar qualificado e na consulta de enfermagem realizar o histórico e o exame físico com uma escuta atenta e sensível a fim de identificar sinais e sintomas de alerta, para o encaminhamento precoce da criança com suspeita de adoecimento(16)

Há relatos que indicaram que famílias buscaram variados serviços públicos e privados e, mesmo utilizando de diversos recursos, o diagnóstico do câncer infantil também foi demorado.

No começo que eu pagava consulta numa clínica, mas não resolveu [...]. Eu fui a muitos hospitais [públicos], em emergência, em UPA e ninguém resolvia [...]. Um dia levei na UPA, lá na baixada e uma médica me disse que era uma coisa muito grave e que era para eu levar ele num hospital grande, porque na baixada não ia resolver. Até que eu levei ele num hospital da zona sul [hospital municipal], que descobriu e mandou ele para cá. (Entrevista 2)

Na época a gente tinha plano de saúde, [...]. Tudo foi feito numa clínica particular [...]. Chegamos a ir para emergência, umas duas ou três vezes, isso já no finalzinho, mas não conseguiam diagnosticar. Lá na clínica, um médico depois de ter feito todos os exames, partiu para um exame mais profundo, foi quando constatou. No mesmo dia encaminharam a gente para cá [centro de tratamento] e já internaram. (Entrevista 10)

Os itinerários baseiam-se em escolhas dos caminhos de tratamento percorridos, a partir das visões de mundo e escolhas realizadas pelos pacientes, sobretudo, em relação ao funcionamento dos serviços de saúde e cuidado recebido. Entretanto, as dificuldades de acesso ao serviço especializado faz com que os familiares definam novas trajetórias na busca por atendimento e esclarecimento diagnóstico ${ }^{(4,6-7)}$, conforme evidenciado nos achados do estudo. 
Essa mesma realidade foi constatada nas vozes de familiares de adolescentes com diabetes que também buscaram atendimento em consultórios privados, planos de saúde e serviços do Sistema Único de Saúde, caracterizando uma rede de diversificados caminhos e orientações. Demostrando assim que as dificuldades enfrentadas por usuários dos serviços de saúde não se limitam ao câncer infantil ${ }^{(7)}$

Logo, nem sempre a família escolhe inicialmente o SUS para buscar o diagnóstico do filho. Três famílias buscaram primeiro atendimento no serviço ambulatorial privado, mas antes da chegada ao centro de tratamento as crianças passaram por unidades públicas de saúde. Nesses casos, o percurso até o diagnóstico não foi demorado.

Primeiro eu fui numa consulta particular que me encaminhou para um hospital municipal aqui no Rio. Ele ficou internado um dia, no outro dia de manhã eles me trouxeram pra cá, conseguiram vaga aqui. (Entrevista 13)

Levei ele num pediatra particular e depois levei ele numa UPA em Botafogo, onde o pediatra suspeitou de câncer. Aí arrumaram uma transferência para cá. Ele ficou um dia internado na UPA e no dia seguinte veio pra cá. (Entrevista 15)

Levei no pediatra do plano dele. Depois no posto, mandaram para um hospital grande. Foi para um hospital municipal e depois me transferiram para cá. (Entrevista 21)

Atualmente, a rede privada oferece uma gama de recursos, serviços e variadas especialidades médicas. Entretanto, nos resultados desta pesquisa as famílias que buscaram os serviços de saúde privados não obtiveram o tratamento nestes serviços e sim nos serviços públicos de saúde. Tais fatos demonstram os problemas e fragilidades tanto do serviço privado quanto do sistema público ${ }^{(17)}$.

Parte das famílias buscou o primeiro atendimento nos serviços de emergência, sejam eles em unidades de pronto-atendimento ou hospitais públicos ou privados. Algumas vezes sem uma resolução rápida, e em outras o diagnóstico e encaminhamento para uma unidade de referência se deu mais rapidamente:

Leveielenuma emergência [Hospital Privado], ficamos 4 dias lá e fomos transferidos para [Hospital Privado] para fazer mielograma, em 24hjátinha dado o resultado. (Entrevista 3)

Levei para UPA e hospital. Quando levei a última vez, ela ficou internada na UPA, de lá mandaram para outro hospital [público] onde ela ficou um mês e duas semanas internadae de lá ela veio para cá [centro de tratamento]. (Entrevista 12)
Levamos na UPA e no dia seguinte já estava aqui [hospital, cenário da pesquisa]. Foi assim um processo muito rápido, porque essa médica da UPA de Cabo Frio por coincidência era uma médica daqui. (Entrevista 20)

Sabe-se também que o diagnóstico de câncer em uma criança é um evento raro, logo um médico clínico-geral, pode ter dificuldades em reconhecer o câncer infantil ${ }^{(18)}$. O mesmo pode acontecer com os enfermeiros que atuam na classificação de risco das emergências e também atendem essas crianças, pois eles, geralmente, identificam as urgências e emergências e dão prioridade aos casos mais graves ${ }^{(19)}$.

Assim, reforça-se a importância de investimento na capacitação dos profissionais, visto que uma grande parte dos pacientes com câncer é diagnosticada após surgimento de manifestações que ameaçam a vida, devido ao câncer não diagnosticado precocemente ${ }^{(2)}$.

Apenas um dos familiares cita que utilizou sistema de regulação online como mecanismo de marcação de consulta, como segue:

Aí eu paguei uma médica que mandou procurar um reumatologista infantil. Tive muita dificuldade para achar um reumatologista infantil. Tive que inscrever no SISREG, porque eu não encontrava. (Entrevista 1)

O encaminhamento do paciente no SUS para atendimento às variadas especialidades e níveis de atenção depende de um sistema de referência e contrarreferência que ter fluxo articulado. Sendo a referência o encaminhamento para os níveis de maior grau ou complexidade e a contrarreferência o retorno do paciente de uma complexidade maior para um nível de complexidade menor, assim, o paciente continua sendo acompanhado pela atenção primária(16).

A partir dos depoimentos dos familiares verificou-se que esse fluxo de referência e contrarreferência não acontece de forma adequada.

Depois levei no ortopedista também não deu nada. Ele piorou e levei ele no PAM, três vezes. Na terceira vez a médica resolveu internar, mas não melhorou. Depois ele veio para cá ser atendido pela reumatologista que encaminhou logo para emergência pediátrica [...]. (Entrevista 6)

Levei ele em vários hospitais, ninguém sabia o que ele tinha, batia raio-x, tudo ninguém sabia o que era. Aí eu fui ao posto de saúde, no posto mesmo me indicaram que eu tinha que levar ele num hospital grande. (Entrevista 21) 
Esse achado, que aponta a inadequação na manutenção do fluxo de atendimento, indica que os estudos sobre itinerários dão suporte para a avaliação da efetividade dos serviços na garantia do acesso e atendimento das necessidades da população. Assim, subsidiam o desenvolvimento de ações educativas em saúde, capacitação profissional e adequação de fluxos ${ }^{(4)}$.

Tem-se ainda a invisibilidade da Estratégia de Saúde da Família (ESF), nos relatos dos familiares, como um recurso público de saúde buscado pela família ou como um serviço de acompanhamento da criança e familiares.

A ESF garante a aproximação dos profissionais e dos centros de saúde da família à comunidade, aumentando a capacidade de resolutividade dos problemas de saúde mais comuns por meio da continuidade do cuidado, o que implica em melhoria da qualidade de vida. Além disso, deve ser o primeiro lugar que a família procura quando houver a suspeita de que algo esteja errado com a criança, sendo fundamental uma capacitação profissional para que seja possível captar as suspeitas de câncer infantil e a rápida referência para o centro de tratamento(20).

Assim, no contexto da ESF, o enfermeiro deve buscar favorecer um olhar atentivo e uma escuta qualificada para as crianças com sinais e sintomas sugestivos de câncer, favorecendo, assim o diagnóstico precoce dessa doença.

\section{口 CONSIDERAÇÕES FINAIS}

Este estudo permitiu que se resgatasse a trajetória percorrida por crianças com câncer, desde os primeiros sinais e sintomas até o diagnóstico e o encaminhamento para a unidade de tratamento especializado.

Ficou evidente que as maiores dificuldades encontradas foram o despreparo dos profissionais médicos em identificar e investigar mais profundamente os sinais e sintomas que a criança apresentava. Além da necessidade de peregrinação pelos diversos serviços de saúde até o alcance do diagnóstico.

Os familiares não fizeram referências específicas ao enfermeiro, contudo, sua atuação, nos diferentes serviços de saúde, pode contribuir significativamente para o diagnóstico precoce do câncer infantil. Logo, reconhece-se a necessidade de políticas públicas, educação permanente e ações efetivas que sejam capazes de capacitar os profissionais de saúde nos variados níveis do SUS e na rede privada com o intuito de diminuir o tempo que decorre da sintomatologia até o diagnóstico da doença.

Conclui-se que para o diagnóstico precoce do câncer infantil e consequentemente maiores chances de cura, são necessárias ações dos serviços de saúde, das institui- ções que formam os profissionais, buscando promover um cuidado integral já que foi possível perceber um comprometimento deste princípio. Os profissionais, entre eles os enfermeiros que atuam nas classificações de risco das emergências e na atenção básica, devem conhecer os sinais e sintomas do câncer infantil e utilizar os recursos que o sistema de saúde dispõe, não esquecendo de olhar o indivíduo em sua totalidade. Além da necessidade do adequado funcionamento dos sistemas de referência e contra referência dos SUS, inclusive como melhor atuação da Estratégia de Saúde da Família.

Como limitação da pesquisa, aponta-se que a mesma retrata a realidade de crianças atendidas em uma instituição do Rio de Janeiro, assim sugere-se realização de novos estudos, em outras regiões do país para ampliação da discussão e comparação destes resultados com outros.

\section{口 REFERÊNCIAS}

1. Ministério da Saúde (BR), Instituto Nacional do Câncer. Estimativa 2018: incidência de câncer no Brasil. Rio de Janeiro; 2017 [citado 2018 fev 16]. Disponível em: http://www.inca.gov.br/estimativa/2016/estimativa-2016-v11.pdf.

2. Ministério da Saúde (BR), Instituto Nacional de Câncer. Diagnóstico precoce do câncer na criança e no adolescente. Rio de Janeiro; 2014 [citado 2016 jun 12]. Disponível em: http://pesquisa.bvsalud.org/brasil/resource/pt/sus-34538.

3. Dommett RM, Redaniel MT, Stevens MCG, Hamilton W, Martin RM. Features of childhood cancer in primary care: a population-based nested case-control study. Br J Cancer. 2012;106(5):982-7. doi: https://doi.org/10.1038/bjc.2011.600.

4. Cabral ALLV, Martinez-Hemáez A, Andrade EIG, Cherchiglia ML. Itinerários terapêuticos: 0 estado da arte da produção científica no Brasil. Ciênc Saúde Coletiva. 2011;16(11):4433-42. doi: https://doi.org/10.1590/S141381232011001200016

5. Fundato CT, PetrilliAS, Dias CG, Gutiérrez MGR. Itinerário terapêutico de adolescentes e adultos jovens com osteossarcoma. Rev Bras Cancerol. 2012;58(2):197-208.

6. Oliveira BRG, Collet N, Mello DF, Lima RAG. The therapeutic journey of families of children with respiratory diseases in the public health service. Rev LatinoAm Enfermagem. 2012;20(3):453-61. doi: https://doi.org/10.1590/S010411692012000300005

7. Finco $M$, Bertoncini JH. Therapeutic itinerary and experience of families and adolescents with type 1 diabetes mellitus. Rev Bras Promoç Saúde. 2016;29(3). doi: https://doi.org/10.5020/18061230.2016.p371.

8. Lima BC. 0 itinerário terapêutico da família da criança com câncer: contribuiç̧ões da enfermagem [monografia]. Niterói (RJ): Escola de Enfermagem, Universidade Federal Fluminense; 2016.

9. Minayo, MCS. 0 desafio do conhecimento: pesquisa qualitativa em saúde. 14. ed. São Paulo: Hucitec; 2014.

10. Ahrensberg JM, Olesen F, Hansen RP, Schrøder H, Vedsted P. Childhood cancer and factors related to prolonged diagnostic intervals: a Danish population-based study. Br J Cancer. 2013;108(6):1280-7. doi: https://doi.org/10.1038/bjc.2013.88.

11. Ahrensberg JM, Fenger-Grøn M, Vedsted P. Use of primary care during the year before childhood cancer diagnosis: a nationwide population-based matched comparative study. PLoS One. 2013;8(3):e59098. doi: https://doi.org/10.1371/ journal.pone.0059098. 
12. Oliveira K, Veronez M, Marques CDC, Higarashi IH, Marcon SS. Route taken by families of children hospitalized in a teaching hospital. Rev Bras Enferm. 2014;67(1):36-42. doi: https://doi.org/10.5935/0034-7167.20140004.

13. França T, Medeiros KR, Belisario SA, Garcia AC, Pinto ICM, Castro IL, et al. Continuous health education policy in Brazil: the contribution of the teaching-service integration standing committees. Ciênc Saúde Coletiva. 2017;22(6):1817-28. doi: https://doi.org/10.1590/1413-81232017226.30272016.

14. Guimarães TM, Silva LF, Santo FHE, Moraes JRMM. Palliative care in pediatric oncology in nursing students' perception. Esc Anna Nery. 2016;20(2):261-7. doi: https://doi.org/10.5935/1414-8145.20160035.

15. Frank BRB, Viera CS, Ross C, Obregón P, Toso BRG0. Avaliação da longitudinalidade em unidades de Atenção Primária à Saúde. Saúde Debate. 2015;39(105):40010. doi: http://dx.doi.org/10.1590/0103-110420151050002008.

16. Ministério da Saúde (BR), Secretaria de Atenção à Saúde, Departamento de Atenção Básica. Política Nacional de Atenção Básica. Brasília (DF): Ministério da Saúde; 2012.
17. Buboltz FL, Silveira A, Neves ET. Strategies for families of children served in pediatric first aid: the search for the construction of integrality. Texto contexto Enferm. 2015;24(4):1027-34. doi: https://doi.org/10.1590/01040707201500002040014.

18. Dommett RM, Redaniel T, Stevens MC, Martin RM, Hamilton W. Risk of childhood cancer with symptoms in primary care: a population-based case-control study. Br J Gen Pract. 2013;63(606):e22-9. doi: https://doi.org/10.3399/ bjgp13X660742.

19. Veras JEGLF, Joventino ES, Coutinho JFV, Lima FET, Rodrigues AP, Ximenes LB. Risk classification in pediatrics: development and validation of a guide for nurses. Rev Bras Enferm. 2015;68(5):913-22. doi: https://doi.org/10.1590/00347167.2015680521i.

20. Malta DC, Santos MAC, Stopa SR, Vieira JEB, Melo EA, Reis AAC. Family Health Strategy coverage in Brazil, according to the National Health Survey, 2013. Ciênc Saúde Coletiva. 2016;21(2):327-38. doi: https://doi.org/10.1590/141381232015212.23602015 .
Autor correspondente:

Liliane Faria da Silva

E-mail: lili.05@hotmail.com
Recebido: 23.02.2018

Aprovado: 02.07.2018 